\title{
Room temperature magnetism of ordered porphyrin layers on Fe
}

Cite as: Appl. Phys. Lett. 115, 082404 (2019); https://doi.org/10.1063/1.5109750

Submitted: 12 May 2019. Accepted: 02 August 2019 . Published Online: 21 August 2019

M. S. Jagadeesh, A. Calloni (D), A. Brambilla (D), A. Picone, A. Lodesani (i), L. Duò, F. Ciccacci (D) M. Finazzi (D), and G. Bussetti (i)
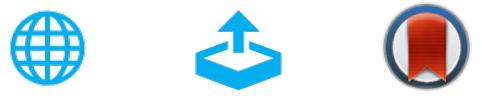

View Online

Export Citation

\section{ARTICLES YOU MAY BE INTERESTED IN}

Hydrogel-mediated semiconductor wafer bonding

Applied Physics Letters 115, 081601 (2019); https://doi.org/10.1063/1.5096540

Substantially enhanced ferroelectricity in $\mathrm{JT}$ ion $\mathrm{Cu}^{2+}$-doped $\mathrm{Co}_{1-\mathrm{x}} \mathrm{Cu}_{\mathrm{x}} \mathrm{Cr}_{2} \mathrm{O}_{4}(\mathrm{O} \leq \mathrm{x} \leq 0.4)$

Applied Physics Letters 115, 082903 (2019); https://doi.org/10.1063/1.5111789

Ultra-wide-bandgap AlGaN homojunction tunnel diodes with negative differential resistance

Applied Physics Letters 115, 082104 (2019); https://doi.org/10.1063/1.5113503

\section{Applied Physics Letters}

Mid-IR and $\mathrm{THz}$ frequency combs special collection 


\title{
Room temperature magnetism of ordered porphyrin layers on Fe
}

\author{
Cite as: Appl. Phys. Lett. 115, 082404 (2019); doi: 10.1063/1.5109750 \\ Submitted: 12 May 2019 . Accepted: 2 August 2019 . \\ Published Online: 21 August 2019
}

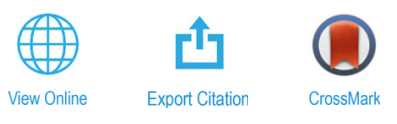

\author{
M. S. Jagadeesh, A. Calloni, ${ }^{\text {a) }}$ (D) A. Brambilla, (D) A. Picone, A. Lodesani, (D) L. Duò, F. Ciccacci, (D) M. Finazzi, \\ and G. Bussetti (D)
}

\author{
AFFILIATIONS \\ Dipartimento di Fisica, Politecnico di Milano, Piazza Leonardo da Vinci 32, 20133 Milano, Italy \\ a)alberto.calloni@polimi.it
}

\begin{abstract}
We propose a method to grow metal tetraphenyl porphyrin (MTPP) molecular layers where a long-range structural and magnetic order can be achieved simultaneously and at room temperature by a proper treatment of the ferromagnetic substrate. We focus in particular on the oxygenpassivated $\mathrm{Fe}(001)-p(1 \times 1) \mathrm{O}$ surface, where MTPP molecules (with $\mathrm{M}=\mathrm{Co}$ and $\mathrm{Ni}$ ) arrange by forming square commensurate overlayers. Spin-resolved photoemission detects a clear spin-splitting of CoTPP electronic states, while no magnetic response is obtained from NiTPP, as expected from the electronic configuration of the respective free molecules. We link these observations to the decoupling action of oxygen at the interface, whose effect is to enhance the molecular diffusivity and tune the electronic interaction with the substrate electronic structure.
\end{abstract}

Published under license by AIP Publishing. https://doi.org/10.1063/1.5109750

Engineering and manipulating ordered arrays of spins is one of the goals of current spintronic research. Once created, an ordered layer of magnetic moments can be used to encode and elaborate information. Given the small size of molecules and relying on their chemical stability, a relatively high information density can be obtained with a potentially negligible energy consumption, provided that a reliable method is found to address each molecule individually for writing/ reading. ${ }^{1-7}$ Porphyrins-heterocyclic compounds where four pyrrole units are arranged to form a macrocycle-are ideal candidates for this kind of application: the planar structure of the macrocycle and the possibility of tuning the chemical nature of the peripheral groups makes it possible to organize such molecules on flat substrates in an ordered fashion, ${ }^{8}$ while the possibility of hosting a metallic ion at the center with unpaired $3 d$ electrons ensures the presence of localized magnetic moments. ${ }^{9,10}$ When chemical reactions occur within those molecules, specific molecular arrangements are induced and both the electronic and magnetic properties are significantly affected. Tuning these properties in view of obtaining a regular assembly of magnetic units is one of the main goals of magnetochemistry. ${ }^{11,12}$ Magnetic ordering at room temperature (RT) and without the need of external magnetic fields is obtained by choosing a ferromagnetic substrate ${ }^{13,14}$ and stems either from the direct exchange between the unpaired electrons of the molecule and the substrate electronic states or from indirect superexchange mediated by the $\mathrm{N}$ ligands. ${ }^{9,15}$ However, the choice of using traditional magnetic substrates, such as transition metals
(TMs), is usually precluded by the poor morphological quality and structural integrity of the molecular layers, due to the strong chemical interaction with the surface. ${ }^{16,17}$ As a result, the magnetic behavior of both the molecules and the surface (magnetic moments, exchange interaction, and surface anisotropy) is affected. ${ }^{18,19}$ Although such a strong interaction might be an advantage in some cases, especially when spin transport across the molecule/metal interface is involved, ${ }^{20,21}$ long range ordering is possible only by achieving a certain degree of decoupling with the substrate, as shown for molecular deposition on lowinteracting, nonmagnetic substrates such as noble metals. ${ }^{22-24}$ We identify in the passivation of the surface a viable strategy for tuning the molecular coupling. ${ }^{25,26}$ Some earlier attempts are acknowledged, where ultrathin oxides $^{27-29}$ or graphene ${ }^{30-32}$ were specifically used to change the nature of the interaction between the molecules and the substrate to indirect superexchange mediated by the interlayer. For most of these systems, however, scarce structural information about the molecular layers is reported.

In this letter, we report an investigation on the magnetic ordering of $\mathrm{Co}$ and $\mathrm{Ni}$ tetraphenyl porphyrins (MTPP, with $\mathrm{M}=\mathrm{Co}$ and $\mathrm{Ni}$, respectively) on $\mathrm{Fe}(001)$. Surface passivation is obtained by dosing $\mathrm{O}$ atoms. This procedure forms a monolayer-thick rock salt oxide (formal 1:1 stoichiometry) where $\mathrm{O}$ atoms occupy the fourfold hollow sites in between the $\mathrm{Fe}$ atoms, resulting in a $p(1 \times 1) \mathrm{O}$ superstructure. After surface passivation, the Fe substrate shows an improved morphology ${ }^{33}$ and enhanced magnetic moments. ${ }^{34}$ We already demonstrated the 
effectiveness of $\mathrm{Fe}(001)$ surface passivation in promoting the long range assembling of a number of molecular species, such as $\mathrm{C}_{60}$ molecules ${ }^{25}$ and nonmagnetic $\mathrm{Zn}$ tetraphenyl porphyrins (ZnTPP). For the latter, taken as representative of low-interacting MTPP having no free coordination sites, we detected the formation of a wetting layer with a $(5 \times 5)$ periodicity, characterized by an electronic structure similar to that of free-standing molecules. ${ }^{17,35,36}$ These results depict a regime of "partial decoupling" with the substrate, characterized by the absence of strong hybridization, which might nevertheless support non-negligible interactions such as those of an exchange type, leading to a ferromagnetic ordering of MTPP magnetic moments. Conversely, the strong interaction between $\mathrm{ZnTPP}$ and bare $\mathrm{Fe}(001)$ is reflected in (i) a loss of the surface order [due to a reduced mobility of the molecules in contact with the substrate and linked to the absence of a low energy electron diffraction (LEED) pattern] and (ii) a quenching of those photoemission features related to the tetrapyrrole ring (see below for more details). ${ }^{17,36}$

Molecular deposition was performed in a ultrahigh vacuum system (base pressure as low as $10^{-8} \mathrm{~Pa}$ ) equipped with dedicated chambers for the substrate preparation and the deposition of the organic layers. ${ }^{37}$ The $\mathrm{Fe}(001)-p(1 \times 1) \mathrm{O}$ substrate was prepared by first depositing a thick (about $500 \mathrm{~nm}$ ) Fe layer on top of $\mathrm{MgO}(001)$ and subsequently dosing $30 \mathrm{~L}$ [ 1 langmuir $(\mathrm{L})=10^{-6}$ Torr $\cdot \mathrm{s}$ ] of molecular oxygen with the surface kept at $450^{\circ} \mathrm{C}$. Excess oxygen was finally removed by flash-heating up to $700^{\circ} \mathrm{C}$. MTPP molecules were evaporated from a $\mathrm{BN}$ crucible held at about $300^{\circ} \mathrm{C}$ and their flux [kept at about 0.3 molecular layers (ML) per minute] was monitored by means of a quartz microbalance. The substrate was kept at RT during molecular evaporation and the subsequent measurements.

LEED and scanning tunneling microscopy (STM) were used to investigate the molecular assembling at the completion of $1.0 \mathrm{ML}$ of either CoTPP or NiTPP on $\mathrm{Fe}(001)-p(1 \times 1) \mathrm{O}$. Figure 1 (a) shows the sharp diffraction pattern observed on the CoTPP monolayer (similar results are obtained for NiTPP). The substrate $(1 \times 1)$ diffraction spots

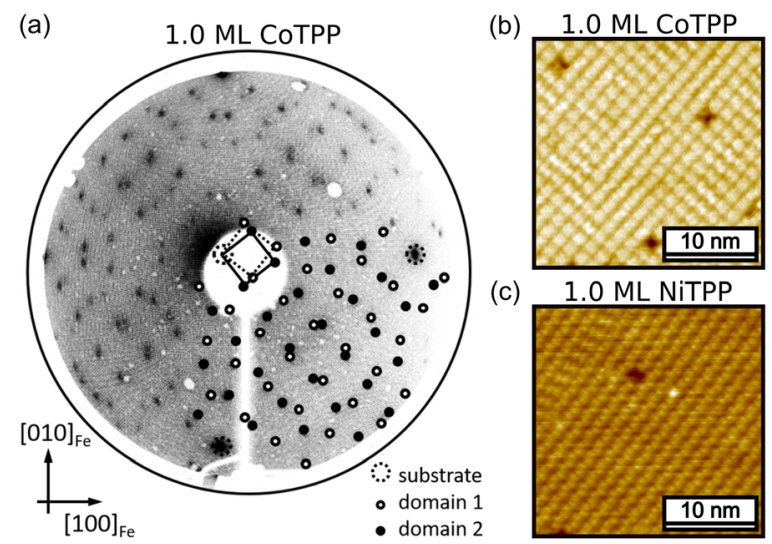

FIG. 1. (a) LEED pattern from 1.0 ML of CoTPP on Fe(001)-p $(1 \times 1) 0$ acquired at a beam energy of $55 \mathrm{eV}$ (the pattern obtained at the same conditions from $1.0 \mathrm{ML}$ of NiTPP on Fe(001)-p $(1 \times 1) 0$ is substantially identical). The white and black dots identify the extra spots related to the molecular lattice, while the dashed circles mark the position of the substrate diffraction spots (see the text for details). (b) and (c) topographic STM images related to the CoTPP and NiTPP ML, respectively. The tunneling parameters are $I=1 \mathrm{~mA}$ and $V=1.4 \mathrm{~V}$ for panel (b) and $I=500 \mathrm{pA}$ and $V=2 \mathrm{~V}$ for panel (c). are highlighted with dashed circles, while the remaining diffraction signal is attributed to the presence of a commensurate molecular superstructure corresponding to a square molecular lattice rotated by $\pm 36.9^{\circ}$ with respect to the substrate $\langle 001\rangle$ directions and with a lattice parameter of $1.43 \mathrm{~nm}$, i.e., the same molecular spacing previously detected for ZnTPP. ${ }^{17}$ The STM topography images reported in Figs. 1(b) and 1(c) are consistent with the diffraction data and clearly show the presence of a uniform molecular layer covering of the $\mathrm{Fe}(001)-p(1 \times 1) \mathrm{O}$ surface (see also the STM image of Fig. S1 in the supplementary material, acquired on a larger area), characterized by extended domains and a relatively small amount of defect sites (molecular vacancies).

The magnetic behavior of CoTPP and NiTPP molecules was investigated by means of spin resolved photoemission and inverse photoemission spectroscopies (SR-PES and SR-IPES, respectively). Both techniques are available in situ for the mapping of the spin-resolved electronic structure of both filled and empty states, as we demonstrated for the $\mathrm{Fe}(001)$ $p(1 \times 1) \mathrm{O}$ substrate in Ref. 37 . Spin resolution in PES is obtained from a mini Mott detector mounted at the exit slit of the photoelectron energy analyzer. ${ }^{38}$ In SR-IPES, we use a GaAs(001) crystal excited with circularly polarized light for the generation of spin-polarized electrons. ${ }^{39}$ The samples were magnetized by applying a pulsed magnetic field ( $>100$ Oe) parallel to the in-plane $[100]_{\mathrm{Fe}}$ easy axis. PES (IPES) spectra were acquired at RT, at magnetic remanence and at normal electron emission (incidence), thus probing only the in-plane component of the electron spin polarization. PES data were analyzed following the strategy of Ref. 38 to remove instrumental asymmetries.

Figure 2(b) shows our results in the 0.0-1.0 ML CoTPP coverage range, together with the spin-integrated spectra from a thicker (4.0 ML) film [Fig. 2(a)], which can be considered as representative of molecules no longer interacting with the substrate. ${ }^{35}$ The PES signal from $\mathrm{Fe}(001)-p(1 \times 1) \mathrm{O}[$ Fig. $2(\mathrm{~b})]$ is characterized by a sharp peak at about $-4.3 \mathrm{eV}$ due to photoemission from $\mathrm{O} 2 p$-derived states and some small intensity signal from bulk Fe $3 d$ states close to the Fermi energy $\left(E_{\mathrm{F}}\right){ }^{40,41}$ Two spin-resolved features are visible on the corresponding IPES spectrum [Fig. 2(b)], due to transitions toward the bulk majority and minority-spin empty states. ${ }^{42}$ At the completion of the CoTPP monolayer, the emission from $\mathrm{O} 2 p$ orbitals is quenched, while a significant contribution still comes from metal $3 d$ states, both for PES and IPES. By comparison with the spectra acquired on the thick film and the available literature on CoTPP, we identify the CoTPP molecular features at ML coverage with a series of broad peaks: the one located at about $-1.6 \mathrm{eV}$ accounts for photoemission from the highest occupied molecular orbital (HOMO), while the lowest unoccupied molecular orbital (LUMO) is expected to contribute to a feature located at about $1.5 \mathrm{eV}$, i.e., partially overlapping with the signal from the bulk Fe states. The shift of the molecular features away from $E_{\mathrm{F}}$ observed with PES (IPES) at 4.0 ML coverage (panel a) is due to a less efficient screening of the photoemission hole (extra electron) by the substrate with respect to the ML configuration. ${ }^{17,36}$ The HOMO and LUMO features are related to electronic states localized on the main tetrapyrrole ring of the CoTPP molecule, while the other features can be either related to photoemission from the ring (label " $\mathrm{R}$ ") or from the four peripheral phenyl groups (labeled " $\mathrm{Ph}$ "). ${ }^{17,43}$ According to this analysis, the spectra from the bare $\mathrm{Fe}(001)-p(1 \times 1) \mathrm{O}$ surface provide only an approximate description of the substrate contribution at ML coverage, a result that we attribute to the combined effect of electronic modifications occurring at the interface and the distortion of 
(a)

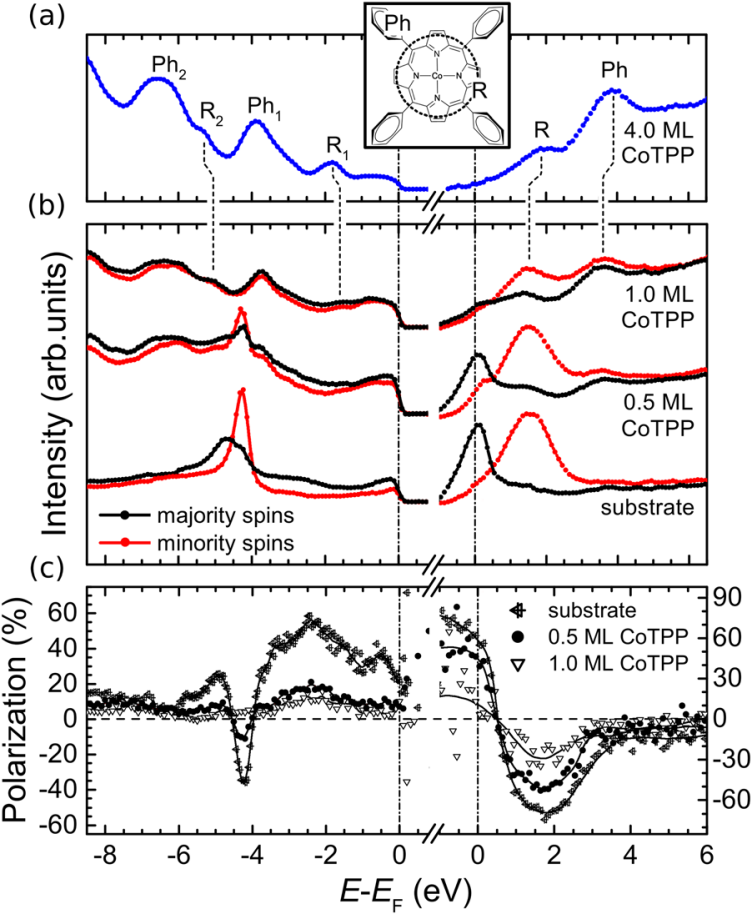

FIG. 2. (a) Spin-integrated photoemission spectra from a $4.0 \mathrm{ML}$ CoTPP/Fe(001)$p(1 \times 1) 0$ sample. Labels "R" or "Ph" are related to photoemission from either the tetrapyrrole ring or the phenyl groups, respectively (see also the schematic drawing of the CoTPP molecule in the inset). (b) Spin-resolved spectra at different coverages in the ML range. (c) Spin polarization $P$ of the spectra in (b), computed as $P=100 \frac{\mathrm{maj}-\mathrm{min}}{\mathrm{maj}-\mathrm{min}}$, where maj (min) stands for the majority (minority) spin spectrum. Continuous lines drawn through the experimental points are a guide to the eye.

the substrate photoemission signal by scattering from the molecular layer, particularly effective when working, as in the present case, with a finite-acceptance spectrometer. ${ }^{44,45}$

The spectral polarization presented in Fig. 2(c) is seen to decrease with increasing CoTPP coverage, a behavior linked to the progressive attenuation of the photoemission signal from the substrate and from the interface. At ML coverage, the Fe contribution is still significant (at least close to $E_{\mathrm{F}}$ ), making it difficult to assess the spin polarization of molecular features without properly taking it into account.

Instead of focusing on the detailed analysis of the molecular electronic states located close to $E_{\mathrm{F}}$, we aim our attention at those molecular states located at a larger binding energy (BE), following to some extent the methodology of Ref. 21. In such an energy region, no features from the substrate are present nor expected and the overall spectral polarization-mainly accounting, as explained before, for the substrate contribution-is lowered below 5\% [Fig. 2(c)]. A similar behavior can be observed also with other MTPP molecules (NiTPP results are reported in Fig. S2 of the supplementary material, as an example). Consequently, with reference to only the photoemission spectra of filled states in Fig. 3, related to 1.0 ML and 4.0 ML CoTPP films, we select the spectral region $\mathrm{R}_{2}\left(\mathrm{Ph}_{2}\right)$ accounting for the ring (phenyl) contribution. The spin-resolved results are presented in the insets after the subtraction of a spin-dependent linear background, accounting for the spin-polarized (featureless) residual contribution from the substrate. We

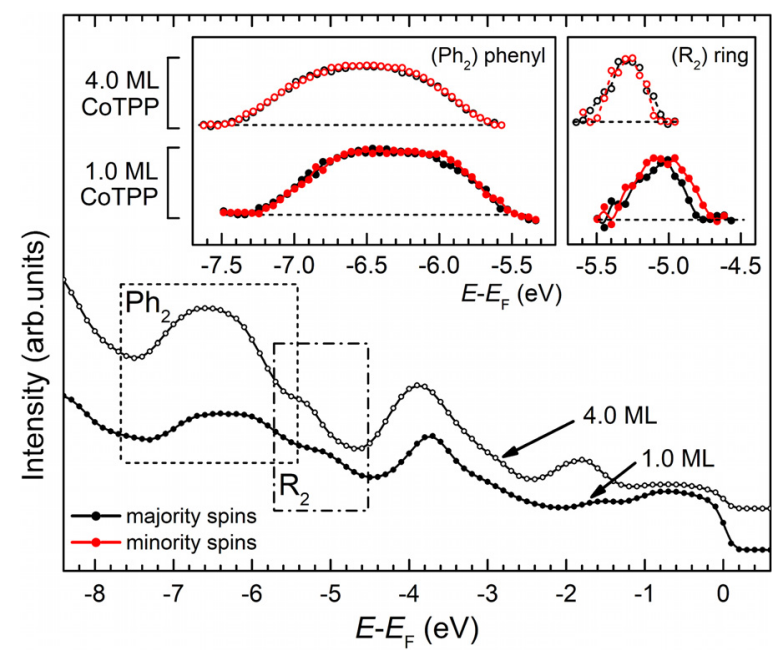

FIG. 3. Main panel: spin-integrated photoemission from a CoTPP/Fe(001)-p $(1 \times 1) 0$ sample at different Co coverages: 1.0 ML (closed symbols) and 4.0 ML (open symbols). The photoemission signal of region $\mathrm{R}_{2}\left(\mathrm{Ph}_{2}\right)$ is related to electronic states prevalently located in the COTPP tetrapyrrole ring (phenyl groups). In the insets: high statistics spin resolved photoemission acquired in regions $\mathrm{R}_{2}$ and $\mathrm{Ph}_{2}$ for both CoTPP coverages. The photoemission spectra are shown after the subtraction of a linear background.

observe that 4.0 ML spectra do not show any spin dependence, as expected considering that relevant magnetic interactions are limited to the molecule/substrate interface. ${ }^{27,46-48}$ Conversely, a significant imbalance between the spin channels is observed at 1.0 ML in the spectra related to region $\mathrm{R}_{2}$, i.e., to electronic states localized on the ring. In particular, we detect a splitting of about $0.1 \mathrm{eV}$ at the low BE side of the ring feature, characterized by a negative polarization. This result was reproducibly observed on several nominally identical samples. Conversely, the same imbalance is not observed in the spectra related to photoemission from peripheral groups (region $\mathrm{Ph}_{2}$ ).

Our experimental results for NiTPP molecules are shown in Fig. 4: compared to CoTPP, we find no significant splitting in region $\mathrm{R}_{2}$ (ring states). ZnTPP molecules were additionally investigated as prototypical not-magnetic molecules, as their $\mathrm{Zn}^{\mathrm{II}}$ ion is characterized by a completely filled $3 d$ shell, thus providing a lower detection limit for our magnetic investigation. We remark that, although with a different epitaxial relationship with the $\mathrm{Fe}(001)-p(1 \times 1) \mathrm{O}$ substrate, ZnTPP arrange on the surface by forming an ordered lattice of flat-lying molecules showing the same packing of $\mathrm{Co}(\mathrm{Ni}) \mathrm{TPP}$. The differences between $\mathrm{Zn}$ and $\mathrm{Co}(\mathrm{Ni}) \mathrm{TPP}$ systems are therefore likely limited to the TM ion. The absence of any spin splitting also in ZnTPP spectra (differences in the photoemission intensity between the two spin channels are within the experimental uncertainty reported in Fig. 4), allows us to rule out any strong contribution coming from the $\mathrm{C}$ - and $\mathrm{N}$ - derived orbitals to the overall magnetic signal of MTPP monolayers.

We can rationalize our $\mathrm{Co}(\mathrm{Ni}) \mathrm{TPP}$ results in the light of the electronic structure of free-standing molecules, ${ }^{9}$ consistent with the small electronic structure perturbation expected from the interaction with the $\mathrm{Fe}(001)-p(1 \times 1) \mathrm{O}$ surface. The magnetic response of such molecules is related to the presence of unpaired spins on the central TM ion, where $3 d$ states are split by a $\mathrm{D}_{4 \mathrm{~h}}$-symmetry crystal field. While CoTPP are characterized by a low-spin $(S=1 / 2)$ ground state, NiTPP 


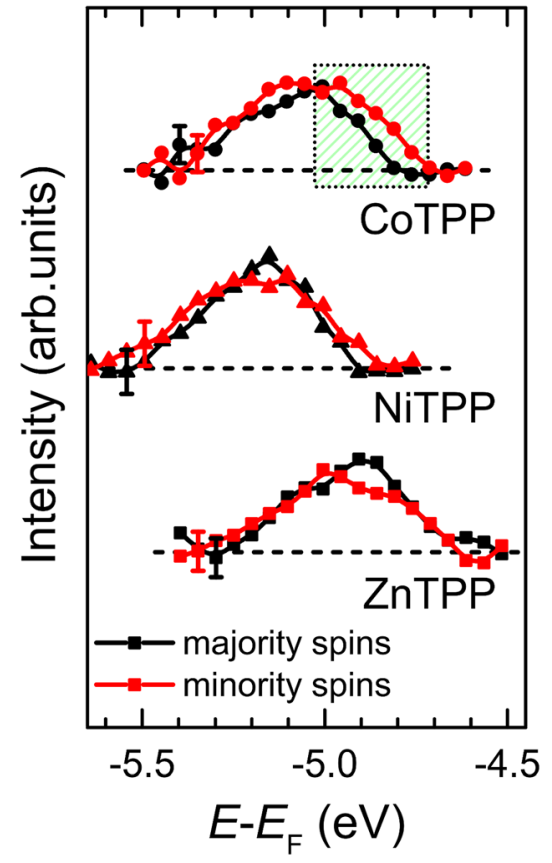

FIG. 4. Spin-resolved photoemission from 1.0 ML of MTPP $(M=C o, N i, Z n)$ on $\mathrm{Fe}(001)-p(1 \times 1) \mathrm{O}$. Spin-polarized spectra have been acquired in region $\mathrm{R}_{2}$ (see Fig. 3), and are displayed after the subtraction of a linear background. Statistical error bars are inserted at the beginning of each spectrum showing the experimental uncertainty. The green square highlights the spectral region in which we observe a negative spectral polarization. This result was reproducibly observed on several nominally identical samples.

are characterized by a singlet $(S=0)$ ground state, ${ }^{10}$ i.e., by a nil magnetic moment.

In conclusion, we characterized a very interesting hybrid magnetic system, namely a single layer of porphyrins grown on an ultrathin Fe oxide layer. We have definitively proven that such a system simultaneously matches the requirements of (i) long-range order, with the formation of a molecular superstructure commensurate with the substrate surface lattice and (ii) spin-dependent response even at room temperature, allowing for its possible use in hybrid molecular devices. The enabling technology here is the passivation of the $\mathrm{Fe}(001)$ substrate, providing the molecules with (i) a sufficient mobility to self-assemble on the surface and (ii) a certain degree of electronic decoupling to retain their free-state magnetic behavior without completely quenching the magnetic interaction with the substrate, as we verified after careful scrutiny of the valence electronic structure of several MTPP molecular species by means of spin-resolved photoemission spectroscopy.

See the supplementary material for a large scale STM image of 1.0 ML CoTPP/Fe $(001)-p(1 \times 1) \mathrm{O}$ (Fig. S1) and the spin polarization of SR-PES spectra for 1.0 ML Co and NiTPP/Fe(001)- $p(1 \times 1) \mathrm{O}$ in the $\mathrm{R}_{2}$ and $\mathrm{Ph}_{2} \mathrm{BE}$ region (Fig. S2).

\section{REFERENCES}

${ }^{1}$ L. Bogani and W. Wernsdorfer, Nat. Mater. 7, 179 (2008).

${ }^{2}$ M. N. Leuenberger and D. Loss, Nature 410, 789 (2001).
${ }^{3}$ S. Thiele, F. Balestro, R. Ballou, S. Klyatskaya, M. Ruben, and W. Wernsdorfer, Science 344, 1135 (2014)

${ }^{4}$ P. Ferriani, S. Heinze, and V. Bellini, Sci. Rep. 7, 3647 (2017).

${ }^{5}$ J. Bartolomé, F. Bartolomé, N. B. Brookes, F. Sedona, A. Basagni, D. Forrer, and M. Sambi, J. Phys. Chem. C 119, 12488 (2015).

${ }^{6}$ G. Bussetti, M. Campione, M. Riva, A. Picone, L. Raimondo, L. Ferraro, C. Hogan, M. Palummo, A. Brambilla, M. Finazzi, L. Duò, A. Sassella, and F. Ciccacci, Adv. Funct. Mater. 24, 958 (2014).

${ }^{7}$ C. Wäckerlin, D. Chylarecka, A. Kleibert, K. Müller, C. Iacovita, F. Nolting, T. A. Jung, and N. Ballav, Nat. Commun. 1, 1 (2010).

${ }^{8}$ L. Grill, M. Dyer, L. Lafferentz, M. Persson, M. V. Peters, and S. Hecht, Nat. Nanotechnol. 2, 687 (2007).

${ }^{9}$ J. M. Gottfried, Surf. Sci. Rep. 70, 259 (2015).

${ }^{10}$ M.-S. Liao and S. Scheiner, J. Chem. Phys. 117, 205 (2002).

${ }^{11}$ L. M. Arruda, M. E. Ali, M. Bernien, F. Nickel, J. Kopprasch, C. Czekelius, P. M. Oppeneer, and W. Kuch, J. Phys. Chem. C 123, 14547 (2019).

${ }^{12}$ N. Ballav, C. Wäckerlin, D. Siewert, P. M. Oppeneer, and T. A. Jung, J. Phys. Chem. Lett. 4, 2303 (2013).

${ }^{13}$ A. Scheybal, T. Ramsvik, R. Bertschinger, M. Putero, F. Nolting, and T. A. Jung, Chem. Phys. Lett. 411, 214 (2005).

${ }^{14}$ H. Wende, M. Bernien, J. Luo, C. Sorg, N. Ponpandian, J. Kurde, J. Miguel, M. Piantek, X. Xu, P. Eckhold, W. Kuch, K. Baberschke, P. M. Panchmatia, B. Sanyal, P. M. Oppeneer, and O. Eriksson, Nat. Mater. 6, 516 (2007).

${ }^{15}$ D. Chylarecka, T. K. Kim, K. Tarafder, K. Müller, K. Gödel, I. Czekaj, C. Wäckerlin, M. Cinchetti, M. E. Ali, C. Piamonteze, F. Schmitt, J.-P. Wüstenberg, C. Ziegler, F. Nolting, M. Aeschlimann, P. M. Oppeneer, N. Ballav, and T. A. Jung, J. Phys. Chem. C 115, 1295 (2011).

${ }^{16}$ H. C. Herper, S. Bhandary, O. Eriksson, B. Sanyal, and B. Brena, Phys. Rev. B 89, 085411 (2014).

${ }^{17}$ G. Bussetti, A. Calloni, M. Celeri, R. Yivlialin, M. Finazzi, F. Bottegoni, L. Duò, and F. Ciccacci, Appl. Surf. Sci. 390, 856 (2016).

${ }^{18}$ K. V. Raman, Appl. Phys. Rev. 1, 031101 (2014).

${ }^{19}$ F. Djeghloul, M. Gruber, E. Urbain, D. Xenioti, L. Joly, S. Boukari, J. Arabski, H. Bulou, F. Scheurer, F. Bertran, P. Le Fèvre, A. Taleb-Ibrahimi, W. Wulfhekel, G. Garreau, S. Hajjar-Garreau, P. Wetzel, M. Alouani, E. Beaurepaire, M. Bowen, and W. Weber, J. Phys. Chem. Lett. 7, 2310 (2016).

${ }^{20}$ M. Cinchetti, V. A. Dediu, and L. E. Hueso, Nat. Mater. 16, 507 (2017).

${ }^{21}$ S. Lach, A. Altenhof, K. Tarafder, F. Schmitt, M. E. Ali, M. Vogel, J. Sauther, P. M. Oppeneer, and C. Ziegler, Adv. Funct. Mater. 22, 989 (2012).

${ }^{22}$ S. Stepanow, A. Mugarza, G. Ceballos, P. Moras, J. C. Cezar, C. Carbone, and P. Gambardella, Phys. Rev. B 82, 014405 (2010).

${ }^{23}$ J. Bartolomé, F. Bartolomé, L. M. García, G. Filoti, T. Gredig, C. N. Colesniuc, I. K. Schuller, and J. C. Cezar, Phys. Rev. B 81, 195405 (2010).

${ }^{24}$ P. Gargiani, M. Angelucci, C. Mariani, and M. G. Betti, Phys. Rev. B 81, 085412 (2010).

${ }^{25}$ A. Picone, D. Giannotti, M. Riva, A. Calloni, G. Bussetti, G. Berti, L. Duò, F. Ciccacci, M. Finazzi, and A. Brambilla, ACS Appl. Mater. Interfaces 8, 26418 (2016).

${ }^{26}$ A. Brambilla, A. Picone, D. Giannotti, A. Calloni, G. Berti, G. Bussetti, S. Achilli, G. Fratesi, M. I. Trioni, G. Vinai, P. Torelli, G. Panaccione, L. Duò, M. Finazzi, and F. Ciccacci, Nano Lett. 17, 7440 (2017).

${ }^{27}$ A. P. Weber, A. N. Caruso, E. Vescovo, M. E. Ali, K. Tarafder, S. Z. Janjua, J. T. Sadowski, and P. M. Oppeneer, Phys. Rev. B 87, 184411 (2013).

${ }^{28}$ D. Klar, B. Brena, H. C. Herper, S. Bhandary, C. Weis, B. Krumme, C. Schmitz-Antoniak, B. Sanyal, O. Eriksson, and H. Wende, Phys. Rev. B 88, 224424 (2013).

${ }^{29}$ D. Chylarecka, C. Wäckerlin, T. K. Kim, K. Müller, F. Nolting, A. Kleibert, N. Ballav, and T. A. Jung, J. Phys. Chem. Lett. 1, 1408 (2010).

${ }^{30}$ C. F. Hermanns, K. Tarafder, M. Bernien, A. Krüger, Y.-M. Chang, P. M. Oppeneer, and W. Kuch, Adv. Mater. 25, 3473 (2013).

${ }^{31}$ G. Avvisati, C. Cardoso, D. Varsano, A. Ferretti, P. Gargiani, and M. G. Betti, Nano Lett. 18, 2268 (2018).

${ }^{32}$ A. Lodesani, A. Picone, A. Brambilla, D. Giannotti, M. S. Jagadeesh, A. Calloni, G. Bussetti, G. Berti, M. Zani, M. Finazzi, L. Duò, and F. Ciccacci, ACS Nano 13, 4361 (2019).

${ }^{33}$ A. Picone, A. Brambilla, A. Calloni, L. Duò, M. Finazzi, and F. Ciccacci, Phys. Rev. B 83, 235402 (2011). 
${ }^{34}$ F. Donati, P. Sessi, S. Achilli, A. Li Bassi, M. Passoni, C. S. Casari, C. E. Bottani, A. Brambilla, A. Picone, M. Finazzi, L. Duò, M. I. Trioni, and F. Ciccacci, Phys. Rev. B 79, 195430 (2009).

${ }^{35}$ A. Picone, D. Giannotti, A. Brambilla, G. Bussetti, A. Calloni, R. Yivlialin, M. Finazzi, L. Duò, F. Ciccacci, A. Goldoni, A. Verdini, and L. Floreano, Appl. Surf. Sci. 435, 841 (2018).

${ }^{36}$ G. Bussetti, A. Calloni, R. Yivlialin, A. Picone, F. Bottegoni, and M. Finazzi, Beilstein J. Nanotechnol. 7, 1527 (2016).

${ }^{37}$ G. Berti, A. Calloni, A. Brambilla, G. Bussetti, L. Duò, and F. Ciccacci, Rev. Sci. Instrum. 85, 073901 (2014).

${ }^{38}$ G. C. Burnett, T. J. Monroe, and F. B. Dunning, Rev. Sci. Instrum. 65, 1893 (1994).

${ }^{39}$ D. T. Pierce, R. J. Celotta, G. -C. Wang, W. N. Unertl, A. Galejs, C. E. Kuyatt, and S. R. Mielczarek, Rev. Sci. Instrum. 51, 478 (1980).

${ }^{40}$ A. Clarke, N. B. Brookes, P. D. Johnson, M. Weinert, B. Sinković, and N. V. Smith, Phys. Rev. B 41, 9659 (1990).

${ }^{41}$ A. Calloni, G. Fratesi, S. Achilli, G. Berti, G. Bussetti, A. Picone, A. Brambilla, P. Folegati, F. Ciccacci, and L. Duò, Phys. Rev. B 96, 085427 (2017).
${ }^{42}$ R. Bertacco and F. Ciccacci, Phys. Rev. B 59, 4207 (1999).

${ }^{43}$ S. Rangan, S. Katalinic, R. Thorpe, R. A. Bartynski, J. Rochford, and E. Galoppini, J. Phys. Chem. C 114, 1139 (2010).

${ }^{44}$ L. Giovanelli, F. C. Bocquet, P. Amsalem, H.-L. Lee, M. Abel, S. Clair, M. Koudia, T. Faury, L. Petaccia, D. Topwal, E. Salomon, T. Angot, A. A. Cafolla, N. Koch, L. Porte, A. Goldoni, and J.-M. Themlin, Phys. Rev. B 87, 035413 (2013).

${ }^{45}$ J. Stöckl, A. Jurenkow, N. Großmann, M. Cinchetti, B. Stadtmüller, and M. Aeschlimann, J. Phys. Chem. C 122, 6585 (2018).

${ }^{46}$ A. Lodi Rizzini, C. Krull, T. Balashov, J. J. Kavich, A. Mugarza, P. S. Miedema, P. K. Thakur, V. Sessi, S. Klyatskaya, M. Ruben, S. Stepanow, and P. Gambardella, Phys. Rev. Lett. 107, 177205 (2011).

${ }^{47}$ G. Avvisati, P. Gargiani, P. Mondelli, F. Presel, A. Baraldi, and M. G. Betti, Phys. Rev. B 98, 115412 (2018).

${ }^{48}$ M. Bernien, X. Xu, J. Miguel, M. Piantek, P. Eckhold, J. Luo, J. Kurde, W. Kuch, K. Baberschke, H. Wende, and P. Srivastava, Phys. Rev. B 76, 214406 (2007). 